FEATURED CASE REPORT

\title{
Long QT syndrome and life threatening arrhythmia in a newborn: molecular diagnosis and treatment response
}

\author{
E Schulze-Bahr, H Fenge, D Etzrodt, W Haverkamp, G Mönnig, H Wedekind, G Breithardt, \\ H-G Kehl
}

Heart 2004;90:13-16

Intrauterine and neonatal manifestations of congenital long QT syndrome are associated with a high cardiac risk, particularly when atrioventricular block and excessive QT prolongation (>600 $\mathrm{ms}^{1 / 2}$ ) are present. In a female newborn with these features, treatment with propranolol and mexiletine led to complete reduction of arrhythmia that was maintained 1.5 years later. High throughput genetic analysis found a sodium channel gene (LQT3) mutation. Disappearance of the 2:1 atrioventricular block and QTc shortening (from $740 \mathrm{~ms}^{1 / 2}$ to $480 \mathrm{~ms}^{1 / 2}$ ), however, was achieved when mexiletine was added to propranolol. This effect was considered to be possibly genotype related. Early onset forms of long QT syndrome may benefit from advanced genotyping.

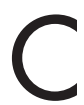
ongenital long QT syndrome (LQTS; prevalence about 1 in 5000) is a primarily familial disease with an autosomal dominant mode of disease transmission. In $12 \%$ of patients with LQTS, sudden death may be the first manifestation of the disease and, importantly, in $4 \%$ it may happen in the first year of life. ${ }^{1}$ The presence of a very long QT interval $(>600 \mathrm{~ms})$, T wave alternans, 2:1 atrioventricular $(\mathrm{AV})$ block, and inner ear deafness are proposed to indicate infants with a high cardiac risk. ${ }^{2}{ }^{3}$ In neonates, LQTS is a rare finding but fetal arrhythmia may already occur during pregnancy. ${ }^{45}$ These observations led to the hypothesis that LQTS may be linked to a subset of sudden infant death syndrome (SIDS). ${ }^{6}$ Recently, several reports finally showed the presence of LQT gene mutations in cases of SIDS or near miss $\operatorname{SIDS}^{78}$ and thereby provided the molecular link between severe arrhythmia syndromes and SIDS. LQTS is a genetically heterogeneous ion channel disorder ${ }^{9}$ in which mutations either in cardiac potassium channel genes or in the cardiac sodium channel gene SCN5A (LQT3) cause the disease. $\beta$ Receptor blocking agents are the treatment of choice for most LQTS patients, potentially even in asymptomatic children. ${ }^{2}$ Sodium channel blockers have specifically been proposed for treatment of the LQT3 subform, since in these mutations addition of sodium channel blockers, mostly but not exclusively in vitro, resulted in a gain of channel function (with a sustained, non-inactivating sodium current that can be suppressed by sodium channel blockade). In the present study, we report on a newborn with congenital LQTS who was severely threatened by perinatal arrhythmias and who was effectively treated after an LQT3 mutation was considered and finally identified.

\section{MATERIAL AND METHODS} Phenotyping

The diagnosis of LQT syndrome was based on common criteria. ${ }^{10}$ Repolarisation features (ST segment and $\mathrm{T}$ wave morphology) in the surface ECG first led to consideration of the LQT3 subtype. Both parents gave written informed consent for genetic analyses. The study was conducted in accordance with the Declaration of Helsinki and recommendations given by the university's local ethics committee.

\section{Genotyping}

Standard methods for genetic analysis were used as previously described. ${ }^{11}$ The BigDye RR Terminator AmpliTaq kit, together with the ABI Prism 3700 Genetic Analyzer (both Perkin-Elmer Applied Biosystems, Weiterstadt, Germany), was used for sequence determination. Nucleic acid deviations were compared with the reference sequence for SCN5A (Genome Database, GI:4506808; GenBank, NM_000335) and confirmed by sequencing of both strands. Identified novel sequence alterations were investigated for their presence in a control group (more than 200 chromosomes $^{11}$ ). When a de novo mutation (not present in either parent) was present in a child, paternity was determined with the AmpFlSTR Profiler Amplification Kit (Perkin-Elmer Applied Biosystems).

\section{RESULTS}

Clinical course of a newborn with congenital LQTS

The female newborn, the first child of non-consanguineous healthy parents (mother's QTc $430 \mathrm{~ms}^{1 / 2}$, father's QTC $410 \mathrm{~ms}^{1 / 2}$ ) (fig 1), was born at week 35 of gestation by caesarean section because of intrauterine episodes of fetal bradycardia and tachycardia. After birth, the baby's Apgar scores were 7 (minute 5) and 9 (minute 10). The neonate had a hydrops fetalis leading to overweight for age (3440 g). Also, a slow heart rate ( 50 beats/min) and cardiopulmonary failure were noted, requiring assisted ventilation for six days. ECG recordings had an extremely long QT interval (QTc $740 \mathrm{~ms}^{1 / 2}$ ) in the setting of a second to third degree $\mathrm{AV}$ block (ventricular rate about 80 beats/min; not shown). Recurrent episodes of non-sustained, polymorphic ventricular tachycardia (torsade de pointes type; 300 beats/min) occurred. At day 1 , the neonate was admitted to the paediatric department; generalised oedema and pleural effusions were present and transthoracic echocardiography showed reduced contractility (fractional shortening $18 \%$ ) of a normal sized left ventricle without signs of structural heart disease. Short runs of torsade de pointes tachycardia reoccurred but electrical defibrillation was not required. Intravenous propranolol was initiated (maximum dosage $4 \mathrm{mg} / \mathrm{kg} / \mathrm{day}$ ) but was only partially effective in suppressing ventricular tachycardia. AV block, however, persisted. Because of the ECG phenotype (long QT interval together with an isoelectric ST segment and

Abbreviations: AV, atrioventricular; LQT3, sodium channel gene; LQTS, long QT syndrome; SIDS, sudden infant death syndrome 

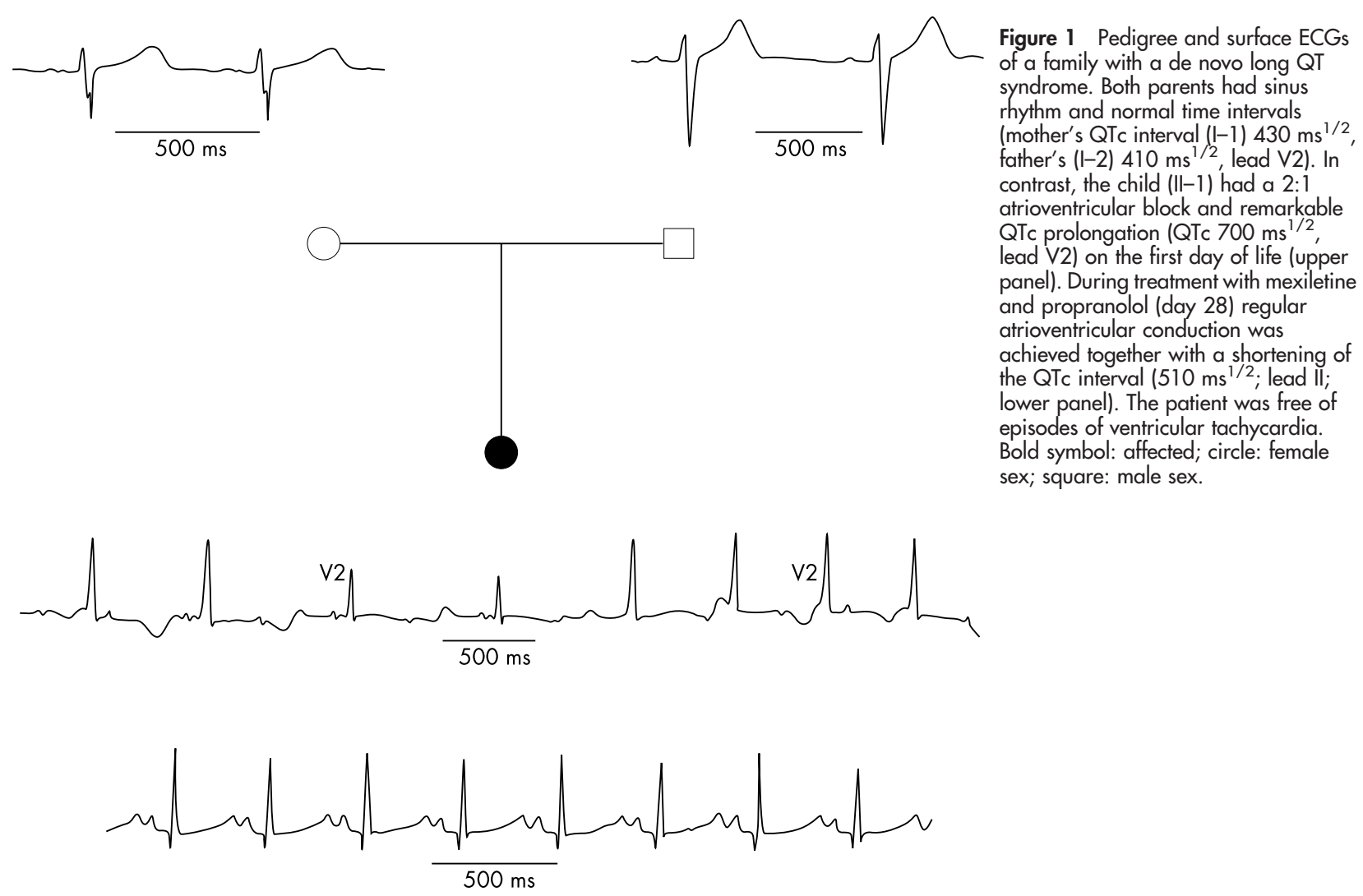

a normal $\mathrm{T}$ wave), the LQT3 subtype was suspected and intravenous mexiletine was started (cumulative dosage at day 1 was $12 \mathrm{mg} / \mathrm{kg} /$ day). To prevent bradycardia related triggering of ventricular arrhythmias, a cardiac pacemaker (VVI mode, 130-110 beats/min) with epimyocardial leads was implanted. Under the cumulated high dosage of mexiletine AV block disappeared and sinus rhythm was established. After day 2, no ventricular tachycardia occurred and QTc was greatly shortened $\left(480 \mathrm{~ms}^{1 / 2}\right)$ (fig 1). Cardiac function improved to normal and the patient was discharged on day 28 (mexiletine $8 \mathrm{mg} / \mathrm{kg} /$ day; propranolol $4 \mathrm{mg} / \mathrm{kg} /$ day). During a follow up period of 1.5 years, the patient remained asymptomatic.

\section{Molecular diagnosis of a LQT3 syndrome}

Because of the ECG phenotype (fig 1), all coding regions of the LQT3 gene SCN5A were first sequenced directly. In exon 23 , a heterozygous $\mathrm{C}$ to $\mathrm{T}$ transversion at nucleotide position 3995 was detected (strand + opposite strand) in the index patient (fig 2). No other mutations were detected in SCN5A. This nucleotide exchange was predicted to cause an amino exchange from proline to leucine at residue 1332 (P1332L; S4-S5 linker of DIII) and is located within a highly conserved protein region (alignment data not shown). In a large control population, the 3995T allele was absent, making a rare polymorphism improbable. Since the mutation (P1332L) was also not identified in both parents (fig 3 ), we considered it to be a de novo mutation after paternity was determined to be very likely. The mutation was indicated by 10 short tandem repeat markers from different chromosomal loci.

\section{DISCUSSION}

In the present case, prenatal episodes of severe arrhythmia were recorded during cardiotocography that required premature birth of a neonate with LQTS. Only a few reports of early onset LQTS are known. Here, we identified a specific sodium channel mutation (P1332L; fig 2) in the setting of an effective, combined medical treatment during follow up.

Fetal manifestations of LQTS may include sinus bradycardia, AV conduction block, and ventricular tachycardia, recordable by magnetocardiography or cardiotocography. ${ }^{3-5}$ Neonates with a very long QT interval $\left(>600 \mathrm{~ms}^{\mathrm{I} / 2}\right)$, a $2: 1$ AV block, or both (as seen in the present case) (fig 1) are at a particularly high cardiac risk ${ }^{2}$ and require immediate and effective treatment. The QTc interval of the present neonate $\left(740 \mathrm{~ms}^{1 / 2}\right)$ is one of the longest reported so far; the observed 2:1 AV block was most likely functional ("pseudo" AV block) because $\mathrm{P}$ waves occurred before the end of the T wave (fig 1 ) and resolved at slower sinus rates (fig 1). Similar cases of LQTS have been reported. ${ }^{4}{ }^{12-16}$. Electrophysiological studies suggested an infra-Hisian location of the block. ${ }^{15}{ }^{16}$ Histomorphological changes of the conduction system are also known in congenital LQTS. ${ }^{17}{ }^{18}$

During high dose treatment with propranolol sinus rhythm was not established in the proband, although this treatment has been shown to be effective in newborns with LQTS. ${ }^{19}$ We questioned whether these different therapeutic responses may be related to different genetic causes of LQTS. ${ }^{20}$ We finally found a heterozygous amino exchange (P1332L) in the SCN5A gene of the proband that suggested that the disease was causative, since, firstly, the mutation was not present in both healthy parents (de novo mutation); secondly; the possibility of a rare polymorphism was determined to be unlikely by investigations of controls; and thirdly, the amino acid residue was evolutionarily conserved (and thereby probably functionally important). Recently, Wedekind et al ${ }^{8}$ identified in an adjacent residue a missense mutation (A1330P) in a patient with neonatally manifesting LQTS who died of a documented cardiac arrest despite high dose propranolol. The specific mutation, in contrast to other LQT3 

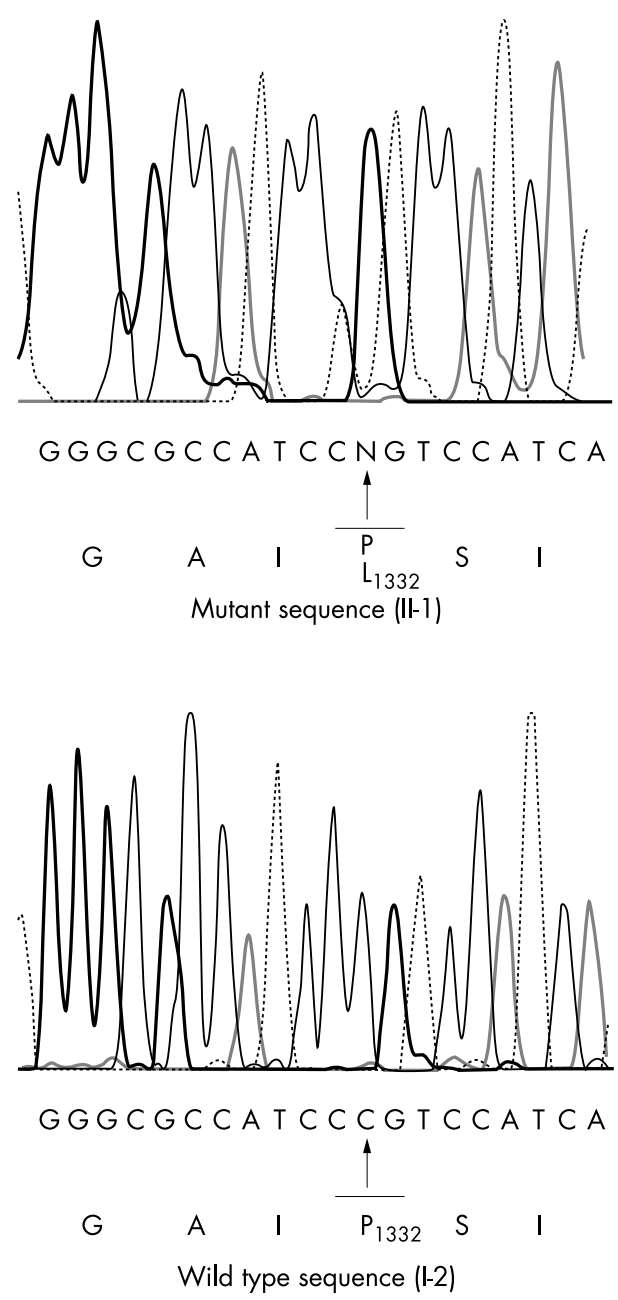

Figure 2 Mutation detection in the SCN5A gene: sequence analysis of exon 23. A heterozygous $C$ to $T$ transversion at nucleotide position 3995 was detected in the index patient (upper part of the panel) and was confirmed by sequencing of the opposite strand. This nucleotide exchange was predicted to cause an amino exchange from proline to leucine at residue 1332 (P1332L). The mutation was not identified in the child's parents. The wild type conformation of the corresponding sequence of exon 23 as detected in the father $(1-2)$ is shown in the lower part of the panel.

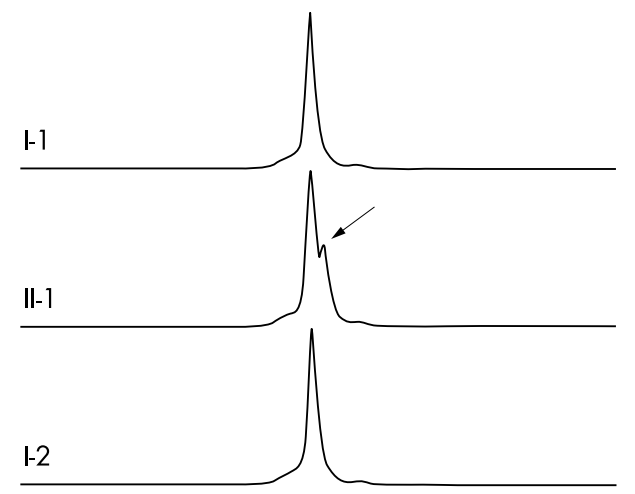

Figure 3 Mutation detection in the SCN5A gene: single strand conformational polymorphism analysis of exon 23 . The configuration was normal (wild type) in the child's parents (I-1 and I-2). In contrast, a migration shiff (arrow) was observed in the child that corresponded to the heterozygous $\mathrm{P} 1332 \mathrm{~L}$ mutation that arose de novo. mutations, augmented the sodium current in vitro by increasing current amplitude (due to acceleration of channel recovery from inactivation and slowing the channel's inactivation). The P1332L mutation may potentially exhibit similar electrophysiological characteristics, but this has not been shown. In contrast to the case reported by Wedekind et $a l_{,}{ }^{8}$ the present neonate was additionally, and potentially successfully, treated with mexiletine, most likely resolving the functional AV block (fig 1) and thereby shortening the QTc interval (from $740 \mathrm{~ms}^{1 / 2}$ to $480 \mathrm{~ms}^{1 / 2}$ ) (fig $\mathrm{l}$ ). Interesingly, pacemaker interrogation further showed that cardiac pacing of the neonate was not needed under this combined treatment.

So far, only limited data are available about neonatal manifestation and treatment of LQTS in relation to specific genotypes. Very recently, Yao and colleagues ${ }^{21}$ reported on a similar case of neonatal LQTS in which a combination of mexiletine and propranolol was effective in suppressing cardiac arrhythmias over a period of two years. Unfortunately, the genotype of this patient was not reported. Conclusions, however, still have to be drawn carefully. We therefore propose that infants with fetal arrhythmias or a postnatal QTc interval $>500 \mathrm{~ms}^{1 / 2}$ during repeated ECG recordings ${ }^{22}$ undergo genetic testing to identify early those with the LQT3 variant, who specifically may benefit from additional sodium blocker, as in the present case. The patient's investigation should include Holter monitoring of the proband and investigation of the family. Even when the parents are unaffected or the family history is unremarkable, immediate genetic testing may be still of value to support the clinical diagnosis and to confirm a de novo onset of the disease that, on the other hand, has implications for the probability of identifying other mutation carriers in the family.

\section{ACKNOWLEDGEMENTS}

The authors are indebted to all of the people who participated in this study. We thank Ellen Schulze-Bahr, Marielies Hesse, Simone Helms, and Susana Pereira for their excellent technical assistance. The present study has been partly supported by the Deutsche Forschungsgemeinschaft (SFB556-Al and Schu 1082/3-9), Bonn, Germany, and the Fondation Leducq, Paris, France.

\section{Authors' affiliations}

${ }^{*}$ E Schulze-Bahr, *W Haverkamp, *G Mönnig, *H Wedekind,

*G Breithardt, Department of Cardiology and Angiology, Hospital of the University of Münster, D-48129 Münster, Germany

D Etzrodt, Molecular Cardiology, Institute for Arteriosclerosis Research at the University of Münster, Domagkstrasse 3, D-48149 Münster, Germany

H Fenge, H-G Kehl, Department of Paediatric Cardiology, University Children's Hospital Münster, D-48129 Münster, Germany

*Also Molecular Cardiology, Institute for Arteriosclerosis Research at the University of Münster

Correspondence to: Dr E Schulze-Bahr, AG Genetics of Arrhythmias, Molekular-Kardiologie, Institut für Arterioskleroseforschung an der Westfälischen Wilhelms-Universität Münster, Domagkstrasse 3, D-48149 Münster, Germany; heart@uni-muenster.de

Accepted 9 June 2003

\section{REFERENCES}

1 Schwartz PJ, Priori SG, Napolitano C. The long QT syndrome. In: Zipes DP, Jalife J, eds. Cardiac electrophysiology: from cell to bedside. Philadelphia: WB Saunders, 2000:597-615

2 Garson AJ, Dick M, Fournier A, et al. The long QT syndrome in children. An international study of 287 patients. Circulation 1993;87: 1866-72.

3 Villain E, Levy M, Kachaner J, et al. Prolonged QT interval in neonates: benign, transient, or prolonged risk of sudden death. Am Heart $J$ 1992; 124:194-7. 
4 Hosono T, Kawamata K, Chiba Y, et al. Prenatal diagnosis of long QT syndrome using magnetocardiography: a case report and review of the literature. Prenat Diagn 2002;22:198-200.

5 Hofbeck M, Ulmer H, Beinder E, et al. Prenatal findings in patients with prolonged QT interval in the neonatal period. Heart 1997;77:198-204.

6 Schwartz PJ. Cardiac sympathetic innervation and the sudden infant death syndrome. A possible pathogenic link. Am J Med 1976;60:167-72.

7 Schwartz PJ, Priori SG, Bloise R, et al. Molecular diagnosis in a child with sudden infant death syndrome. Lancet 2001;358:1342-3.

8 Wedekind H, Smits JP, Schulze-Bahr E, et al. De novo mutation in the SCN5A gene associated with early onset of sudden infant death. Circulation 2001; 104:1158-64.

9 Schulze-Bahr E, Haverkamp W, Borggrefe M, et al. Molecular genetics of arrhythmias: a new paradigm. Z Kardiol 2000;89(suppl 4):IV12-22.

10 Schwartz PJ, Moss AJ, Vincent GM, et al. Diagnostic criteria for the long QT syndrome. An update. Circulation 1993;88:782-4.

11 Schulze-Bahr E, Haverkamp W, Wiebusch H, et al. Molecular analysis at the Harvey Ras-1 gene in patients with long QT syndrome. J Mol Med 1995:73:565-9.

12 Pruvot E, De Torrente A, De Ferrari GM, et al. Two-to-one AV block associated with the congenital long QT syndrome. J Cardiovasc Electrophysiol 1999;10:108-13.

13 Saoudi N, Bozio A, Kirkorian G, et al. Atrioventricular block, and sudden death in the newborn: an electrophysiologic evaluation. Eur Heart $J$ $1991 ; 12: 838-41$.
14 Perticone F, Canepa SA, Ceravolo R, et al. A case of torsade de pointes occurring in a newborn with persistent 2:1 atrioventricular block. Cardiology 1993;83:134-40.

15 Van Hare GF, Franz MR, Roge $C$, et al. Persistent functional atrioventricular block in two patients with prolonged QT intervals: elucidation of the mechanism of block. Pacing Clin Electrophysiol 1990;13:608-18.

16 Lupoglazoff JM, Cheav T, Baroudi G, et al. Homozygous scn5a mutation in long-QT syndrome with functional two-to-one atrioventricular block. Circ Res 2001;89:e 16-21.

17 Bharati S, Dreifus L, Bucheleres $G$, et al. The conduction system in patients with a prolonged QT interval. J Am Coll Cardiol 1985;6:1110-9.

18 Pellegrino A, Ho SY, Anderson RH, et al. Prolonged QT interval and the cardiac conduction tissues. Am J Cardiol 1986;58:11112-3.

19 Stramba-Badiale M, Goulene K, Schwartz PJ. Effects of beta-adrenergic blockade on dispersion of ventricular repolarization in newborn infants with prolonged QT interval. Am Heart J 1997;134:406-10.

20 Priori SG. Gene specific therapy for cardiac disease: the case of long QT syndrome. Rev Port Cardiol 1998;17(suppl 3):III27-38.

21 Yao CT, Wang JN, Tsai YC, et al. Congenital long QT syndrome with functionally impaired atrioventricular conduction: successful treatment by mexiletine and propranolol. J Formos Med Assoc 2002;101:291-3.

22 Schwartz PJ, Garson A Jr, Paul T, et al. Guidelines for the interpretation of the neonatal electrocardiogram. A task force of the European Society of Cardiology. Eur Heart J 2002;23:1329-44.

\section{IMAGES IN CARDIOLOGY}

\section{Calcium channel blocker induced gum hypertrophy: no class distinction}

A 49 year Afro-Caribbean man, with a 10 year history of resistant hypertension, was referred for further management on the following medications: amlodipine $20 \mathrm{mg}$, atenolol $200 \mathrm{mg}$, and enalapril $60 \mathrm{mg}$ daily. Other treatments comprised: two-weekly modecate injections, procyclidine, and nocturnal temazepam $10 \mathrm{mg}$ for stable schizophrenia. He had acquired a degree of renal impairment (creatinine clearance of $64 \mathrm{ml} / \mathrm{min}$ ) as a result of his hypertension, but was not actively requiring dialysis. Pronounced gum hypertrophy with bleeding was a key initial clinical finding (below left). Withdrawal of the dihydropyridine calcium channel blocker resulted in slow regression of the gum hypertrophy. The blood pressure continued to be poorly controlled despite the use of six different antihypertensive drug classes ( $\beta$ blocker, $\alpha$ blocker, angiotensin II receptor blocker, potassium sparing diuretic as well as a loop diuretic, and a centrally acting agent). A non-dihydropyridine

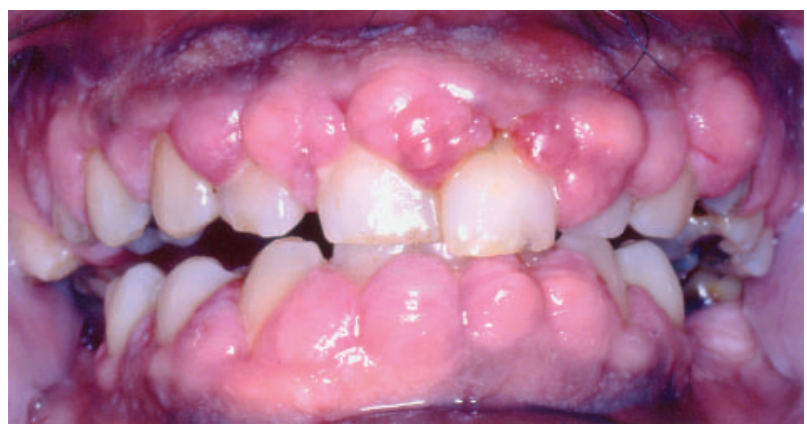

calcium channel blocker (diltiazem XL $240 \mathrm{mg}$ daily) was therefore prescribed to try to improve the blood pressure. Unfortunately the gum features worsened again over a period of three months. They resolved several months after calcium channel blocker withdrawal (below right).

Gum hypertrophy is a well recognised side effect of dihydropyridine calcium channel blockers, with few reports following non-dihydropyridine calcium channel blockers. This case illustrates that it may occur with both major classes of calcium channel blockers and resolve following their cessation.

Y P Samarasinghe A Cox M D Feher yohan.samarasinghe@chelwest.nhs.uk

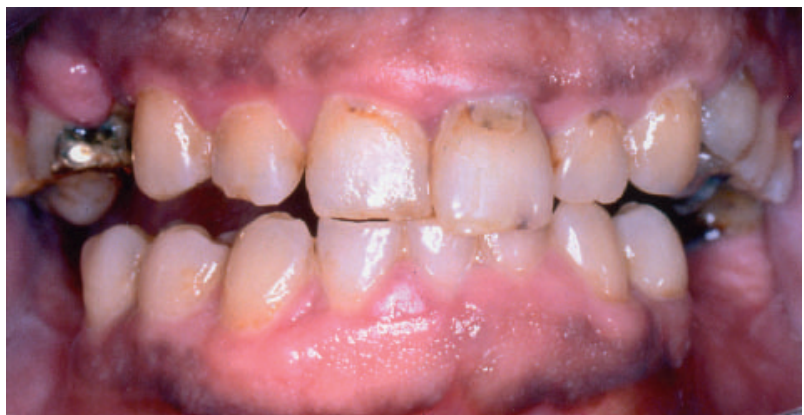

